Ann. Génét. Sél. anim., I976, 8 (3), 343-356.

\title{
OPTIMISATION DU RENOUVELLEMENT DES FEMELLES DANS LES TROUPEAUX LAITIERS SOUMIS AU CROISEMENT TERMINAL
}

\author{
J.-M. ELSEN et J.-C. MOCQUOT \\ Station de Génétique quantitative et appliquée, \\ Centre national de Recherches zootechniques, I. N.R. A., \\ 78350 Jouy en Josas
}

\section{RÉSUMÉ}

Nous avons comparé deux méthodes de choix des femelles traites à soumettre au croisement dans le cas d'une population de vaches laitières ayant un taux de renouvellement minimum. La première méthode considérée permet d'espérer le progrès génétique maximum pour la production laitière puisque seules les femelles ayant un indice laitier élevé sont retenues pour assurer le renouvellement. Cependant, afin d'éviter des difficultés de vêlage, il faut dans ce cas utiliser sur les jeunes femelles non retenues et soumises au croisement des taureaux à viande de plus petit format.

La seconde méthode consiste à exploiter au maximum les possibilités de croisement en ne le pratiquant que sur des femelles âgées avec des taureaux de grand format, alors que toutes les filles des jeunes femelles sont conservées pour le renouvellement.

Ces deux situations ont été comparées sur la base des recettes actualisées qu'elles permettent d'espérer du fait de la vente des veaux croisés et purs d'une part, du supplément de production laitière dâ au progrès génétique d'autre part.

Cette comparaison a été effectuée pour diverses valeurs des taux de renouvellement, du progrès génétique annuel pour la production laitière, des intervalles de génération et du taux d'actualisation, en prenant comme situation de référence la première méthode de choix des femelles.

Outre l'intérêt que présente en soi la démarche utilisée pour le décompte des recettes, son application à chaque combinaison des valeurs envisagées pour les paramètres a permis d'établir des abaques qui fournissent directement le résultat de la comparaison pour une situation particulière en fonction du rapport des prix du lait et de la viande. Dans les conditions actuelles de prix en France, il semble que la seconde solution soit préférable bien qu'elle soit délicate à mettre en œuvre.

\section{IN'TRODUC'TION}

Une politique optimale de renouvellement des femelles traites soumises au croisement terminal doit être définie non seulement par le taux de renouvellement pratiqué mais aussi par le choix des femelles dont on gardera les filles pour former 
la génération suivante, ou, ce qui revient au même, par le choix des femelles à mener en croisement. L'intérêt d'une telle politique doit être jugée à la fois sur ses répercussions à court terme liées au niveau global des productions ou recettes disponibles à un instant donné - et à long terme par son influence sur les possibilités de progrès génétique. Ces deux aspects pouvant être contradictoires (SyRSTAD, 1972), on est conduit à recommander :

- d'abaisser le taux de renouvellement au minimum, en maintenant éventuellement une élimination physique des très mauvaises productrices, de façon à pouvoir choisir les mères des génisses de remplacement et à profiter des effets de l'âge sur la production laitière (ANDERSSON et LINDHE, 1972) ;

- de pratiquer sur toutes les femelles non retenues le croisement avec des mâles de race à viande (ANDERSSON et LINDHE, I972; CunNINGHAM et MCCLINTOCK, I974).

Dans cette hypothèse de taux de renouvellement minimum, le choix des femelles à mener en croisement doit être raisonné sur ses répercussions à court et long terme. A court terme ce choix dépend des différences d'ordre économique entre veaux croisés issus de génisses et ceux issus de vaches adultes, ces différences étant dues à la fois au poids de naissance et aux difficultés de vêlage. Il est alors certain que les recettes seront maximales si toutes les jeunes vaches sont retenues pour le renouvellement, le croisement n'étant pratiqué que sur les adultes. A long terme, le progrès génétique est maximum si le choix des reproductrices se fait par troncature unique sur la valeur génétique estimée par index de ces femelles (BICHARD et al., I973). Cette méthode conduit à ne retenir qu'une partie des génisses pour le renouvellement, les autres étant menées en croisement, et on se place alors vis-à-vis des recettes à court terme, dans une situation sous-optimale. On peut donc se demander si la plusvalue en progrès génétique due à ce type de choix des reproductrices compense la diminution des recettes immédiates et avec quels délais.

Nous nous proposons ici d'étudier ces deux situations. Après les avoir décrites de façon détaillée, nous en étudierons les conséquences sous le double aspect de la production laitière et de la production de viande.

\section{I. - MÉTHODES}

\section{A. - Description des situations}

a) Progrès génétique laitier maximum.

Sous l'hypothèse de taux de croisement maximum, le pourcentage de femelles à inséminer en race pure est fixé par le taux de renouvellement (TR) et par la probabilité qu'une femelle inséminée en race pure donne une génisse de remplacement $\left(p_{r}\right)$. La contribution maximale des femelles, ainsi inséminées, au progrès génétique laitier sera obtenu par un choix sur leur index laitier, en tenant compte de l'écart entre les valeurs génétiques moyennes de chaque classe d'âge (BICHARD et coll., I973).

Si $\Delta g^{\prime}$ est le progrès génétique annuel et $\sigma g$ l'écart-type génétique de la production par lactation dans la population, on supposera que les index laitiers des femelles en $j$ eme lactation ont une distribution normale d'espérance : $-(j-\mathrm{I}) \Delta g^{\prime}$ et d'écart-type $: \sqrt{\mathrm{CD}_{j}} \cdot \sigma g$, où $\mathrm{CD}_{j}$ est le coefficient de détermination de ces index, c'est-à-dire la corrélation entre la valeur génétique vraie et l'index, qui augmente avec le nombre de lactations connues. 
Un choix optimal des mères consiste alors en une troncature unique sur ces différentes distributions d'index.

$\mathrm{Si}$ on désigne par $\alpha j$ la proportion de vaches en $j$ eme lactation et par $q_{j}^{\prime}$ le taux de sélection pratiqué parmi ces vaches, le point de troncature $k^{\prime}$ est tel que pour tout $j$ :

$$
q_{j}^{\prime}=\mathrm{I}-\Pi\left(\frac{k^{\prime}+(j-\mathrm{I}) \Delta g^{\prime}}{\sqrt{\mathrm{CD}_{j}} \cdot g}\right)
$$

où II est la fonction de répartition de la loi normale réduite.

Dès lors, si $\mathbf{N}$. représente la valeur la plus élevée de $j$ :

$$
\sum_{j=1}^{\mathrm{N}} q_{j}^{\prime} \alpha j=\mathrm{TR} / p_{r}
$$

Pour cette solution on supposera que toutes les femelles non retenues pour le renouvellement sont croisées et que, du fait des difficultés de vêlage probables, deux types de mâles de croisement sont nécessaires : mâles de petit format pour les premiers et deuxièmes vêlages, mâles de grand format pour les vêlages suivants.

\section{b) Utilisation optimale du croisement.}

Cette solution, extrême par rapport à la précédente, consiste à rechercher une production maximum de viande par croisement en ne croisant que des vaches ayant vêlé deux fois avec des taureaux de grand format. Elle présente l'avantage de ne faire intervenir qu'un seul type de taureau à viande à sélectionner mais implique par ailleurs de retenir, pour le renouvellement des femelles laitières, tous les produits femelles des premiers et deuxièmes vêlages sans élimination et les filles des meilleures mères en troisième lactation et plus sélectionnées selon un procédé analogue à celui décrit dans la première solution (voir fig. r). Il en résulte un progrès génétique moindre pour la production laitière. Pour la production de viande, le même nombre de veaux croisés est obtenu mais tous sont issus de pères de grand format et de mères âgées. En contrepartie, davantage de veaux mâles de race pure laitière seront issus de mères jeunes et présenteront éventuellement un poids à la naissance inférieur.
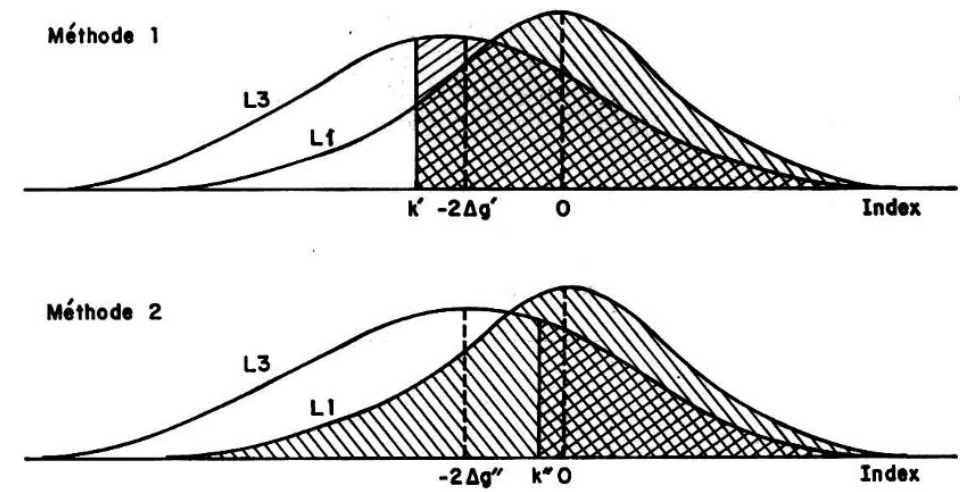

FIG. I. - Méthodes de choix des femelles sur leur index pour le renouvellement Methods for choosing females on their index for replacement

On n'a représenté ici que les distributions des index des femelles en première et troisième lactations. Les zones hachurées correspondent aux femelles retenues pour le renouvellement : avec la méthode $I$, les femelles dont l'index est supérieur à $\mathbf{k}^{\prime}$; avec la méthode 2 , toutes les génisses et femelles en deuxième lactation ainsi que les vaches âgées d'index supérieur à $\mathrm{k}^{\prime \prime}$.

Avec cette solution, l'index minimum $k^{\prime \prime}$ au-delà duquel toutes les femelles en troisième lactation et plus sont retenues pour le renouvellement est tel que, pour tout $j \geqslant 3$ :

et

$$
q_{j}^{\prime \prime}=\mathbf{I}-\Pi\left(\frac{k^{\prime \prime}+(j-\mathbf{I}) \Delta g^{\prime \prime}}{\sqrt{\mathrm{CD}_{j} \sigma g}}\right)
$$

$$
\alpha_{1}+\alpha_{2}+\sum_{j=3}^{N} q_{j}^{\prime \prime} \alpha_{j}=\mathrm{TR} / \mathrm{P}_{r}
$$




\section{B. - Choix des paramètres}

Par hypothèse la première solution a été considérée comme situation initiale et on a cherché à déterminer la perte en lait et le gain en viande consécutifs à la mise en place de la deuxième solution. Cette hypothèse sera discutée ultérieurement. Dans tous les cas nous avons envisagé trois taux de renouvellement : 20,25 et $30 \mathrm{p}$. Ioo et pour chacun d'eux utilisé les paramètres démographiques cités par ANDERSSON et LINDHE (1972), notamment pour les pyramides d'âges et pour la probabilité qu'une IA fécondante fournisse une génisse de renouvellement.

Du point de vue de la sélection, les coefficients de détermination des index laitiers des femelles compte tenu de leur numéro de lactation ont été calculés d'après la méthode française de calcul de ces index (Colleau et Poutous, 1973). On a supposé en particulier des héritabilités de 0,3 et $0,2 \mathrm{I}$ respectivement en première lactation et suivantes. Nous avons par ailleurs admis qu'au moment des inséminations pour le deuxième vêlage, et $a$ fortiori pour le premier, les femelles n'étaient connues que sur ascendance. Le choix des femelles à inséminer pour le renouvellement, donc la voie mères-fille de transmission des gènes, a été déterminé pour chaque solution compte tenu de trois possibilités pour le gain génétique annuel : 30,40 et $50 \mathrm{~kg}$ de lait par an, et deux éventualités pour la somme des intervalles de génération des trois autres voies : I 8 et 23 ans, ce qui correspond grossièrement à une durée d'utilisation des mâles testés sur descendance de I et 3 ans.

Enfin du point de vue économique, gains et pertes ont été comptabilisés sur une durée de I 5 ans avec des taux d'actualisation $(\theta)$ de 0 , ro et 20 p. roo. Une seule durée d'actualisation ayant été envisagée, il nous a en effet semblé utile de donner au taux d'actualisation une plage de variation assez large puisque ces deux paramètres (durée et taux d'actualisation) ont un effet inverse sur le niveau des gains et des pertes actualisées.

\section{C. - Analyse des répercussions à long terme}

\section{- Pertes en lait.}

Le progrès génétique annuel pour la production laitière s'écrit (RENDEL et RoBERTSON, 1950) $\Delta g=\frac{\Sigma \Delta_{i}}{\Sigma L_{t}}$ où $\Delta_{i}$ est la supériorité génétique des reproducteurs de la voie $i$ et $L_{i}$ l'intervalle de génération correspondant, le progrès génétique se transmettant par les voies père-fils, père-fille, mère-fils et mère-fille $(i=\mathrm{I}$ a 4$)$. Dans cette étude, seuls les termes $\Delta_{4}$ et $\mathrm{L}_{4}$ (voie mère-fille) varient et nous noterons $\Delta g^{\prime}=\frac{\Delta_{123}+\Delta_{4}^{\prime}}{\mathrm{L}_{123}+\mathrm{L}_{4}^{\prime}}$ le progrès génétique annuel lié à l'application de la première solution et $\Delta g^{\prime \prime}=\frac{\Delta_{123}+\Delta_{4}^{\prime \prime}}{\mathrm{L}_{123}+\mathrm{L}_{4}^{\prime \prime}}$ pour la seconde solution.

$$
\begin{aligned}
& \Delta_{4}=\frac{\sum_{j=1}^{\mathrm{N}} \alpha_{j} q_{j}^{\prime} i\left(q_{j}^{\prime}\right) \sqrt{\mathrm{CD}_{j}} \sigma g}{\sum_{j=1}^{\mathrm{N}} \alpha_{j} q_{j}^{\prime}} \\
& \mathbf{L}_{4}^{\prime}=a+\frac{\sum_{j=2}^{\mathrm{N}}(j-\mathrm{I}) \alpha_{j} q_{j}^{\prime}}{\sum_{j=1}^{\mathrm{N}} \alpha_{j} q_{j}^{\prime}}
\end{aligned}
$$

où $a$ est l'âge au premier vêlage

$$
\begin{gathered}
\Delta_{4}^{\prime \prime}=\frac{\sum_{j=3}^{\mathrm{N}} \alpha_{j} q_{j}^{\prime \prime} i\left(q_{j}^{\prime \prime}\right) \sqrt{\mathrm{CD}_{j}} \sigma_{g}}{\sum_{j=1}^{\mathrm{N}} \alpha_{j} q_{j}^{\prime \prime}} \\
\mathrm{L}_{4}^{\prime \prime}=a+\frac{\alpha_{2}+\sum_{j=3}^{\mathrm{N}}(j-\mathrm{I}) \alpha_{j} q_{j}^{\prime \prime}}{\alpha_{1}+\alpha_{2}+\sum_{j=3}^{\mathrm{N}} \alpha_{j} q_{j}^{\prime \prime}}
\end{gathered}
$$


La première solution étant considérée comme la situation initiale, on a calculé les taux de sélection $q_{j}^{\prime}$ et la somme des supériorités $\Delta_{123}$ par approximations successives en fonction du progrès génétique initial $\Delta g^{\prime}$ et de la somme des intervalles de génération $\mathrm{L}_{\mathbf{1 2 3}}$ à l'aide des équations (r), (2), (5) et (6). On en a déduit de la même façon le progrès génétique $\Delta g^{\prime \prime}$ et les taux de sélection $q_{j}^{\prime \prime}$ correspondant à la deuxième solution, à l'aide des équations (3), (4), (7) et (8).

\section{- Gain en viande.}

La deuxième solution permet par rapport à la première d'obtenir uniquement des veaux croisés de grand format avec les effectifs relatifs figurés au tableau I

TABLEAU I

Proportion des deux types de veaux croisés

Percentage of the two types of crossed calves

\begin{tabular}{|c|c|c|c|}
\hline & \multicolumn{3}{|c|}{ Nombre d'IA } \\
\hline Solution & $\begin{array}{l}\text { Veaux croisés issus } \\
\text { de mères jeunes } \\
\left.\text { (1er et } 2^{\text {e }} \text { vêlage }\right)\end{array}$ & $\begin{array}{l}\text { Veaux croisés issus } \\
\text { de mères adultes } \\
\text { (3e vêlage et plus) }\end{array}$ & Total \\
\hline 1 & $\left(1-q_{1}^{\prime}\right) \alpha_{1}+\left(1-q_{2}^{\prime}\right) \alpha_{2}$ & $\sum_{j=3}^{N}\left(1-q_{j}^{\prime \prime}\right) \alpha_{j}$ & $1-\mathrm{TR} / \mathrm{P}_{r}$ \\
\hline 2 & 0 & $\sum_{j=3}^{N}\left(1-q_{j}^{\prime \prime}\right) \alpha_{j}$ & $1-\mathrm{TR} / \mathrm{P}_{r}$ \\
\hline
\end{tabular}

Les effectifs réels seront obtenus en multipliant chaque expression par E.P $P_{c}$ où $E$ est l'effectif de la population femelle concernée et $P_{c}$ la probabilité qu'une femelle inséminée en croisement donne un descendant abattu, probabilité supposée indépendante de l'âge de la mère et du type génétique du père. Chaque année, la $2^{\mathrm{e}}$ solution fournit un supplément de $\mathrm{E} . \mathrm{P}_{c}\left(\alpha_{1}\left(\mathrm{I}-q_{1}^{\prime}\right)+\right.$ $\left.\alpha_{2}\left(I-q_{2}^{\prime}\right)\right)$ veaux croisés issus de vaches âgées. De la même façon, si $P_{m}$ est la probabilité qu'une jeune femelle inséminée en race pure donne un mâle abattu, le nombre de ces mâles augmentera de E.P. $P_{m}\left(\alpha_{1}\left(\mathrm{I}-q_{1}^{\prime}\right)+\alpha_{2}\left(\mathrm{x}-q_{2}^{\prime}\right)\right)$ dans la deuxième solution. Par la suite on fera I'hypothèse que $P_{m}=P_{c} / 2$.

\section{D. - Analyse des répercussions à court terme}

Le passage de la situation initiale (première solution) à la situation finale (état d'équilibre correspondant à la deuxième solution) ne se fait pas de façon instantanée : du fait du chevauchement des générations, on observera des fluctuations dans la valeur des taux de sélection optimaux à pratiquer avant d'arriver à des valeurs stables. On peut étudier ces fluctuations par le modèle décrit par Hill (1974) et Elsen et MocQuot (1974).

$$
\mathrm{X}(t)=\mathrm{D}(t) \times \mathrm{X}(t-\mathrm{I})+\mathrm{V}(t)
$$

où $\mathrm{X}(t)$ est un vecteur dont les composantes sont les valeurs génétiques moyennes des mâles et des femelles de chaque classe d'âge à la date $t$

- $\mathrm{D}(t)$, une matrice de transition permettant de passer de la date $t$ - I à la date $t$, dont les éléments sont fonctions des taux de sélection $q_{j}^{t}$ et du mode d'utilisation des reproducteurs.

- $\mathrm{V}(t)$, un vecteur dont les composantes sont les supériorités, à la date $t$ des animaux sélectionnés de chaque classe d'âge par rapport aux candidats à la sélectioł. 
Les taux de sélection $q_{j}^{\prime \prime}$ s seront calculés à partir des relations suivantes, où $\mathrm{X}_{j}^{t}$ est la valeur génétique moyenne des femelles en $j$ eme lactation l'année $t$ :

$$
\begin{gathered}
q_{j}^{\prime \prime t}=\mathrm{I}-\Pi\left(\frac{k+\mathrm{X}_{j}^{t-1}-\mathrm{X}_{3}^{t-1}}{\sqrt{\mathrm{CD}_{j} \sigma g}}\right) \text { pour } j=3 \text { à } \mathrm{N} \\
\alpha_{1}+\alpha_{2}+\sum_{j=3}^{\mathrm{N}} q_{j}^{\prime \prime t} \alpha_{j}=\mathrm{TR} / p_{r}
\end{gathered}
$$

$\mathrm{T}$ étant la durée d'actualisation, on obtiendra alors un ensemble de valeurs $\mathrm{X}(\mathrm{I}) \mathbf{X}(2) \ldots \ldots . \mathrm{X}(\mathrm{T})$ et $q_{j}^{\prime \prime}(\mathrm{r}) q_{j}^{\prime \prime}(2) \ldots \ldots q_{j}^{\prime \prime}(\mathrm{T})$ à partir desquelles on pourra estimer la diminution du progrès génétique en lait et l'augmentation du gain en viande lié à la mise en place de la deuxième politique de conduite des femelles.

\section{- Perte en lait.}

En appelant $\delta(t)$ la différence y $(t)-\mathrm{x}(t)$ entre la valeur génétique moyenne $\mathrm{y}(t)$ des femelles âgées de o à $\mathrm{I}$ an l'année $t$ dans le cas de la première solution $\left(\mathrm{y}(t)=\mathrm{y}(t-\mathrm{r})+\Delta g^{\prime}\right)$ et celle $\mathbf{x}(t)$ de ces mêmes femelles dans le second cas, on peut dresser le tableau 2 suivant, donnant année par année la perte en lait correspondant à chaque numéro de lactation (on a supposé que le premier vêlage avait lieu à 30 mois et que les vêlages suivants intervenaient tous les 12 mois). $d$ est le coefficient d'actualisation $\frac{\mathbf{I}}{\mathrm{I}+\theta}$

\section{TABLEAU 2}

Diminution du progrès génétique laitier dù à la mise en place de la deuxième politique

\begin{tabular}{|c|c|c|c|c|c|c|}
\hline \multirow{2}{*}{$\begin{array}{l}\text { Numéro } \\
\text { de lactation }\end{array}$} & \multicolumn{5}{|c|}{ Année } & \multirow{2}{*}{$\begin{array}{l}\text { Pourcentage } \\
\text { des femelles }\end{array}$} \\
\hline & 12 & 3 & $4 \ldots . \quad N+2$ & $N+3$ & $\ldots \ldots \quad \mathrm{T}$ & \\
\hline 1 & & $\delta(1)$ & $\delta(2) \ldots \delta \delta(N)$ & $\delta(N+1)$ & $\ldots \delta(\mathrm{T}-2)$ & $\alpha_{1}$ \\
\hline 2 & & & $\delta(1) \ldots \delta(N-1)$ & $\delta(\mathrm{N})$ & $\cdots \delta(T-1)$ & $\alpha_{2}$ \\
\hline - & & & - & . & . & . \\
\hline$\dot{0}$ & & & - $\quad \cdot$ & $\dot{0}$ & & - \\
\hline $\mathbf{N}$ & & & $\delta(1)$ & & $\delta(\mathrm{T}-\mathrm{N}-1)$ & $\alpha_{N}$ \\
\hline $\begin{array}{c}\text { Coefficient } \\
\text { d'actualisation }\end{array}$ & $d^{1} d^{2}$ & $d^{3}$ & $\ldots \ldots d^{N+2}$ & $d^{\mathrm{N}+3}$ & $\ldots \ldots d^{T}$ & \\
\hline
\end{tabular}

Decreased genetic progress in milk yield setting up the second method

- Gain en viande.

Ce gain sera donné chaque année par la valeur de :

$$
\begin{gathered}
\text { E.P }\left\{\left(\mathrm{I}-q_{1}^{\prime}\right) \alpha_{1}+\left(\mathrm{I}-q_{2}^{\prime}\right) \alpha_{2}\right\} \\
\text { E. }- \text { Remarques }
\end{gathered}
$$

L'application de cette méthode à toutes les situations envisagées aboutit à un grand nombre de calculs. On a constaté en pratique que la nouvelle valeur du progrès génétique laitier est pratiquement atteinte dès la seconde itération, ce qui semble traduire les fluctuations initiales très limitées de la valeur génétique moyenne des femelles d'une année à la suivante. 
La figure 2 consécutive, à l'étude complète de l'une des éventualités envisagées $\mathrm{TR}=25 \mathrm{p}$. Ioo, $\Delta g=50 \mathrm{~kg}$ et $\mathrm{L}_{128}=\mathrm{I} 8 \mathrm{ans}$ ) illustre cet état de fait. On peut alors, sans perdre trop de précision, assimiler les effets à court terme au seul décalage initial I entre la valeur

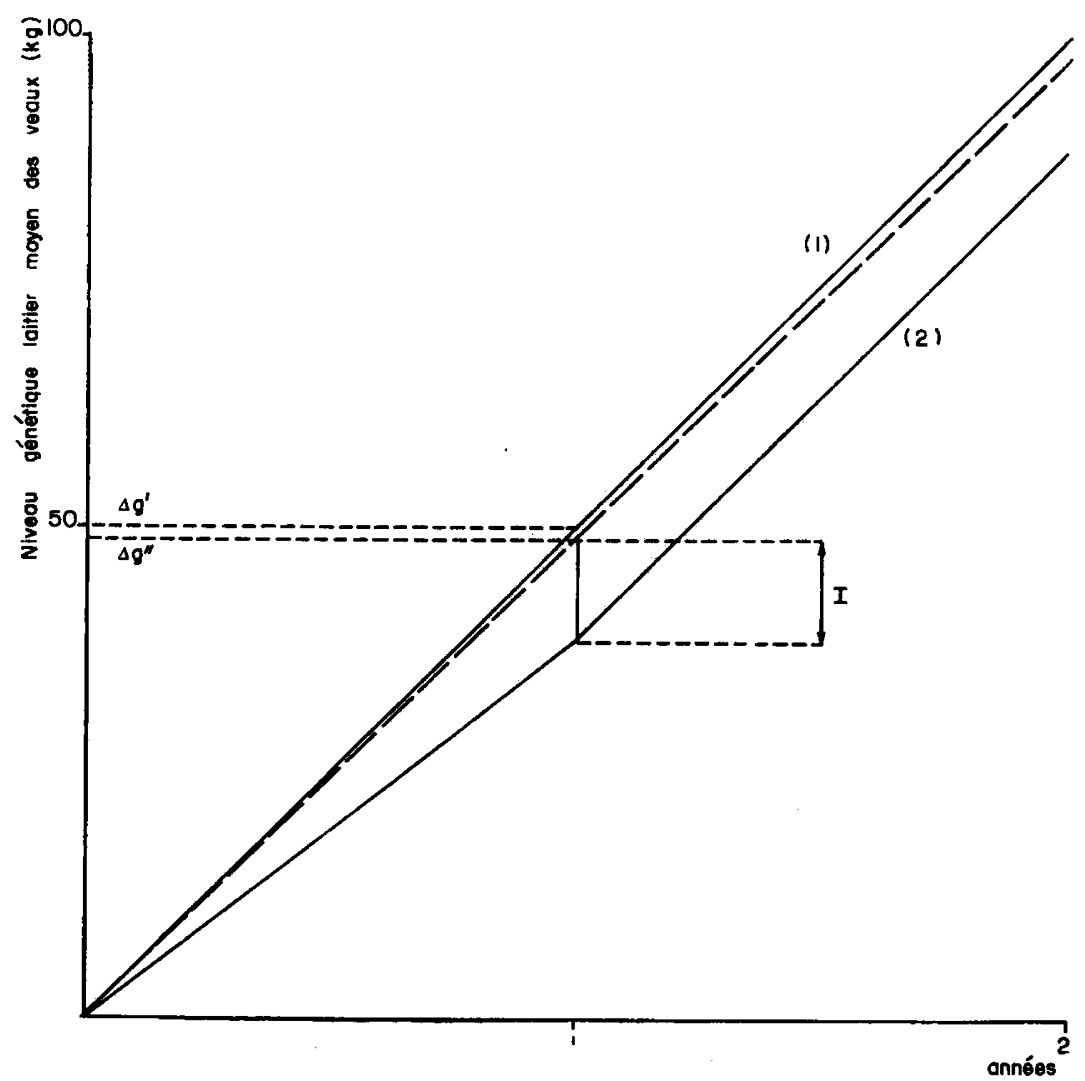

FIG. 2. - Évolution du niveau génétique moyen (base o, l'année o) des veaux femelles pour chacune des deux solutions

Mean genetic level (base o, year o) of female calves for each of the two solutions

est la perte non cumulative due à la mise en place de la seconde solution. $\Delta g^{\prime}$ et $\Delta g^{\prime \prime}$ les progrès génétiques annuels. La perte totale en lait liée à l'applications de la deuxième solution est donnée par la surface comprise entre les courbes (I) et (2).

qu'auraient dû avoir les femelles la première année et celle qu'elles ont effectivement du fait de la mise en place de la seconde solution, puisque ce décalage permanent est atteint dès la première année. Dès lors la perte totale en lait, liée à la seconde solution peut simplement être estimée par la somme des pertes cumulatives.

$$
\mathrm{L}_{1}=\mathrm{E}\left(\Delta g^{\prime}-\Delta g^{\prime \prime}\right) \sum_{j=1}^{\mathrm{N}}\left(\alpha_{j} \sum_{t=j+2}^{\mathrm{T}}(t-j-\mathrm{I}) d^{t}\right)
$$

et des pertes non cumulatives :

$$
\mathrm{L}_{2}=\text { E.I. } \sum_{j=1}^{\mathrm{N}}\left(\alpha_{j} \sum_{t=j+2}^{\mathrm{T}} \mathrm{d}^{t}\right)
$$


Expressions dans lesquelles, I représente la valeur du décalage initial lié à la mise en place de la seconde solution et telle que :

$$
\mathrm{I}=\frac{\Delta_{4}^{\prime}-\Delta_{4}^{\prime \prime}}{2}-\Delta g^{\prime} \frac{\mathrm{L}_{4}^{\prime}-\mathrm{L}_{4}^{\prime \prime}}{2}
$$

Dans ce cas le gain en viande sera exprimé par l'effectif suivant de veaux croisés :

$$
\mathrm{V}=\mathrm{E} . \mathrm{P}_{c}\left(\left(\mathrm{r}-q_{1}^{\prime}\right) \alpha_{1}+\left(\mathrm{I}-q_{2}^{\prime}\right) \alpha_{2}\right) \sum_{t=1}^{\mathrm{T}} \mathrm{d}^{t}
$$

\section{II. — RÉSULTATS}

\section{A. - Résultats physiques}

Pour chaque valeur du taux de renouvellement, du gain génétique initial et de la somme $\mathrm{L}_{128}$ des intervalles de génération des trois voies non concernées, on a calculé la diminution $\Delta g^{\prime}-\Delta g^{\prime \prime}$ de progrès génétique annuel pour la quantité de lait, l'écart initial I de production laitière dû à la mise en place de la deuxième solution, et l'accroissement de la fréquence du croisement sur vaches adultes $\left(\mathbf{r}-q_{1}^{\prime}\right)$ $\alpha_{1}+\left(\mathrm{I}-q_{2}^{\prime}\right) \alpha_{2}$. Les résultats figurent au tableau 3 , ils indiquent les différences entre les deux stratégies étudiées et appellent trois types de remarques.

En premier lieu un progrès génétique laitier initial élevé, et surtout un taux de renouvellement grand réduisent les différences entre les deux solutions. En effet des valeurs élevées de ces deux paramètres tendent pour chacune des deux situations, à accroître la contribution relative des jeunes femelles au renouvellement. On notera d'ailleurs que cette contribution est dans tous les cas élevée, puisqu'au minimum $70 \mathrm{p}$. roo des jeunes femelles sont retenues pour le renouvellement.

En second lieu, et pour ce qui concerne les pertes de production laitière, on remarquera que la différence de progrès génétique annuel reste toujours très faible par comparaison avec la perte initiale de mise en place de la deuxième solution qui est dix fois supérieure et pratiquement indépendante de l'intervalle de génération.

Enfin la proportion supplémentaire d'animaux croisés issus de mères adultes n'est pas affectée par des variations de $\mathrm{L}_{123}$ mais diminue de façon notable quand le progrès génétique laitier augmente du fait d'une utilisation plus large des jeunes femelles pour le renouvellement, dans la première solution. Par ailleurs, la différence entre les deux solutions est réduite du tiers quand le taux de renouvellement passe de 20 à 30 p. I00, les pertes laitières étant dans ce cas trois fois moindres. On remarquera cependant que ces différences marginales sont négligeables vis-à-vis de la réduction considérable du nombre d'animaux croisés consécutive à un taux de renouvellement croissant.

\section{B. - Résultats économiques}

Pour une comparaison plus réaliste des deux situations étudiées nous avons calculé, dans chaque cas, les pertes laitières et les gains en viande cumulés sur I5 ans et actualisés à des taux de 0 , ro et 20 p. 100, selon les expressions simplifiées de $I_{1}$, $\mathrm{L}_{\mathbf{2}}$ et $\mathrm{V}$ citées précédemment. Les valeurs obtenues pour $\mathrm{L}_{\mathbf{1 2 8}}=23$ ans figurent 


\section{TABLEAU 3}

Diminution du progrès génétique laitier

et accroissement de la fréquence du croisement avec des taureaux de grand format lors du passage de la première d la seconde solution

Decreased genetic progress in milk yield and increased frequency of crossing with large-sized bulls during transition from the first to the second solution

\begin{tabular}{|c|c|c|c|c|c|}
\hline \multicolumn{6}{|c|}{ Taux de renouvellement ( 20 p. 100$)$} \\
\hline Critère & $\begin{array}{r}\text { Progrès gêné } \\
\Delta g^{\prime}(\mathrm{kg}\end{array}$ & $\begin{array}{l}\text { que initial } \\
\text { lait) }\end{array}$ & 30 & 40 & 50 \\
\hline \multirow{2}{*}{$\begin{array}{l}\text { Perte en lait } \\
(\mathrm{kg})\end{array}$} & $\mathrm{L}_{123}=18$ ans & $\Delta g^{\prime}-\Delta g^{\prime \prime}$ & $\begin{array}{l}2,35 \\
25,7\end{array}$ & $\begin{array}{c}2,01 \\
22,0\end{array}$ & $\begin{array}{c}1,71 \\
18,7\end{array}$ \\
\hline & $\mathrm{L}_{123}=23$ ans & $\Delta g^{\prime}-\Delta g^{\prime \prime}$ & $\begin{array}{l}1,91 \\
25,8\end{array}$ & $\begin{array}{c}1,64 \\
22,0\end{array}$ & $\begin{array}{r}1,4 \\
18,9\end{array}$ \\
\hline $\begin{array}{l}\text { Gain en viande } \\
\text { (voir texte) }\end{array}$ & \multicolumn{2}{|c|}{$\left(1-q_{1}^{\prime}\right) \alpha_{1}+\left(1-q_{2}^{\prime}\right) \alpha_{2}$} & 0,13 & 0,12 & 0,10 \\
\hline \multicolumn{6}{|c|}{ Taux de renouvellement (25 p. 100) } \\
\hline \multirow{2}{*}{$\begin{array}{l}\text { Perte en lait } \\
\quad(\mathbf{k g})\end{array}$} & $\mathrm{L}_{123}=18$ ans & $\Delta g^{\prime} \underset{\mathrm{I}}{-} \Delta g^{\prime \prime}$ & $\begin{array}{l}1,46 \\
16\end{array}$ & $\begin{array}{l}1,24 \\
13,6\end{array}$ & $\begin{array}{c}1,06 \\
11,6\end{array}$ \\
\hline & $\mathbf{L}_{123}=23$ ans & $\Delta g^{\prime}-\Delta g^{\prime \prime}$ & $\begin{array}{c}1,21 \\
16,1\end{array}$ & $\begin{array}{c}1,01 \\
13,6\end{array}$ & $\begin{array}{c}0,88 \\
11,7\end{array}$ \\
\hline $\begin{array}{l}\text { Gain en viande } \\
\text { (voir texte) }\end{array}$ & \multicolumn{2}{|c|}{$\left(1-q_{1}^{\prime}\right) \alpha_{1}+\left(1-q_{2}^{\prime}\right) \alpha_{2}$} & 0,11 & 0,10 & 0,09 \\
\hline \multicolumn{6}{|c|}{ Taux de renouvellement ( 30 p. 100$)$} \\
\hline \multirow{2}{*}{$\begin{array}{l}\text { Perte en lait } \\
\quad(k g)\end{array}$} & $L_{123}=18$ ans & $\Delta g^{\prime}-\Delta g^{\prime \prime}$ & $\begin{array}{l}0,83 \\
9,0\end{array}$ & $\begin{array}{l}0,71 \\
7,9\end{array}$ & $\begin{array}{l}0,60 \\
6,8\end{array}$ \\
\hline & $\mathrm{L}_{123}=23$ ans & $\Delta g^{\prime}-\Delta g^{\prime \prime}$ & $\begin{array}{l}0,68 \\
9,0\end{array}$ & $\begin{array}{l}0,59 \\
7,9\end{array}$ & $\begin{array}{l}0,51 \\
6,8\end{array}$ \\
\hline $\begin{array}{c}\text { Gain en viande } \\
\text { (voir texte) }\end{array}$ & \multicolumn{2}{|c|}{$\left(1-q_{1}^{\prime}\right) \alpha_{1}+\left(1-q_{2}^{\prime}\right) \alpha_{2}$} & 0,08 & 0,07 & 0,06 \\
\hline
\end{tabular}

$\mathrm{L}_{128}$ est la somme des intervalles de génération des voies de transmission du progrès génétique autres que mère-fille.

$\Delta g^{\prime}-\Delta g^{\prime \prime}$ est la diminution du progrès génétique annuel laitier.

I est la perte non cumulative en lait (voir le texte).

$\left(1-q_{1}^{\prime}\right) \alpha_{1}+\left(1-q_{2}^{\prime}\right) \alpha_{2}$ est l'accroissement de la fréquence du croisement avec des taureaux de grand format. 
au tableau 4. Sans revenir sur les variations de ces valeurs avec le taux de renouvellement ou le progrès génétique initial, on notera que le décalage initial de production laitière intervient pour 65 à 75 p. Ioo de la perte totale en lait selon le taux d'actualisation considéré, - les pertes à long terme étant relativement plus pénalisées par des taux élevés -

\section{TABLEAU 4}

Valeurs totales actualisées par femelle présente des pertes cumulatives et non cumulatives en lait ainsi que du nombre supplémentaire de veaux issus de taureaux. Le grand format quand on passe de la première à la seconde solution

Total discounted values per female of cumulative and non-cumulative milk losses, and of the number of extra calves from large-sized bulls during transition from the first to the second solution

\begin{tabular}{|c|c|c|c|c|c|c|c|c|c|c|}
\hline \multirow{4}{*}{\multicolumn{2}{|c|}{$\begin{array}{c}\text { Taux de } \\
\text { renouvellement } \\
\text { TR }\end{array}$}} & \multicolumn{9}{|c|}{ Progrès génétique $\Delta g$} \\
\hline & & \multicolumn{3}{|c|}{$30 \mathrm{~kg}$} & \multicolumn{3}{|c|}{$40 \mathrm{~kg}$} & \multicolumn{3}{|c|}{$50 \mathrm{~kg}$} \\
\hline & & \multicolumn{9}{|c|}{ Taux d'actualisation } \\
\hline & & $\begin{array}{c}20 \\
\text { p. } 100\end{array}$ & $\begin{array}{c}10 \\
\text { p. } 100\end{array}$ & $\begin{array}{c}0 \\
\text { p. } 100\end{array}$ & $\begin{array}{c}20 \\
\text { p. } 100\end{array}$ & $\begin{array}{c}10 \\
\text { p. } 100\end{array}$ & $\begin{array}{c}0 \\
\text { p. } 100\end{array}$ & $\begin{array}{c}20 \\
\text { p. } 100\end{array}$ & $\begin{array}{c}10 \\
\text { p. } 100\end{array}$ & $\begin{array}{c}0 \\
\text { p. } 100\end{array}$ \\
\hline \multirow{3}{*}{20 p. 100} & $L^{\prime} 1\left({ }^{1}\right)$ & 16 & 40 & 117 & 14 & 35 & 100 & 12 & 29 & 86 \\
\hline & $L^{\times} 2\left({ }^{1}\right)$ & 54 & 107 & 267 & 46 & 92 & 228 & 39 & 79 & 196 \\
\hline & $\mathrm{V}^{\prime}{ }^{(\mathbf{2})}$ & 0,53 & $\mathbf{0 , 8 7}$ & 1,71 & 0,48 & 0,78 & 1,55 & 0,43 & 0,71 & 1,39 \\
\hline \multirow{3}{*}{25 p. 100} & $L^{\prime} 1\left({ }^{1}\right)$ & 11 & 28 & 81 & 10 & 23 & 67 & 8 & 20 & 59 \\
\hline & $L^{\prime} 2\left({ }^{1}\right)$ & 35 & 72 & 175 & 29 & 61 & 147 & 25 & 52 & 128 \\
\hline & $V^{\prime}\left({ }^{2}\right)$ & 0,46 & 0,75 & 1,48 & 0,42 & 0,68 & 1,33 & 0,37 & 0,61 & 1,20 \\
\hline \multirow{3}{*}{30 p. 100} & $L^{\prime} 1\left({ }^{1}\right)$ & 7 & 17 & 48 & 6 & 15 & 42 & 5 & 13 & 36 \\
\hline & $L^{\prime} 2\left({ }^{1}\right)$ & 21 & 43 & 102 & 19 & 38 & 90 & 16 & 32 & 78 \\
\hline & $\mathrm{V}^{\prime}{ }^{\left({ }^{2}\right)}$ & 0,36 & 0,58 & 1,14 & 0,32 & 0,52 & 1,03 & 0,29 & 0,47 & 0,92 \\
\hline
\end{tabular}

(1) En kg de lait actualisé par femelle.

(2) En nombre de veaux actualisé par femelle.

$L^{\prime} 1$ est la perte cumulative en lait ( $L^{\prime} 1=L 1 / E$, voir texte).

$L^{\prime} 2$ est ta perte non cumulative en lait ( $L^{\prime} 2=L 2 / E$, voir texte).

$\mathrm{V}^{\prime}$ est le gain de viande lié à l'accroissement du nombre de veaux croisés issus de taureaux de grand format $\left(V^{\prime}=V / E\right.$, voir texte).

D'un point de vue strictement économique le choix entre les deux solutions doit faire intervenir les marges bénéficiaires par unité de chacun des produits physiques. En particulier si $m \mathrm{~L}$, représente la marge par litre de lait, $m \mathrm{~V}_{1}$ la différence de valeur des veaux croisés issus de jeunes femelles et de vaches adultes et $m \mathrm{~V}_{2}$ 
cette même différence pour les veaux mâles de race pure, la première solution sera préférable à la seconde si $\left(\mathrm{L}_{1}+\mathrm{L}_{12}\right) m \mathrm{~L}$ est supérieur à $\mathrm{V}\left(m \mathrm{~V}_{1}-\frac{m \mathrm{~V}_{2}}{2}\right)$. Dès lors les deux solutions peuvent être comparées par la confrontation du rapport des marges : $m=\frac{\left(m \mathrm{~V}_{1}-\frac{m \mathrm{~V}_{\mathrm{s}}}{2}\right)}{m \mathrm{~L}}$ et du rapport des produits physiques cumulés actualisés; $P=\frac{L_{1}+L_{2}}{V}$. La première solution est préférable quand $P$ est supérieur à $m$. On trouvera sur la figure 3 les différentes valeurs de ce rapport $P$ en fonction des variables du modèle (progrès génétique initial, taux de renouvellement, somme des intervalles de générations $L_{123}$ et taux d'actualisation). Cette figure peut être utilisée comme une abaque pour déterminer la meilleure solution. Par exemple, pour un rapport des marges $m$ de 167 , la seconde solution sera toujours préférée si le taux de renouvellement est de $30 \mathrm{p}$. roo, la première si le taux d'actualisation est de o p. roo et le taux de renouvellement 20 p. $x$ oo.

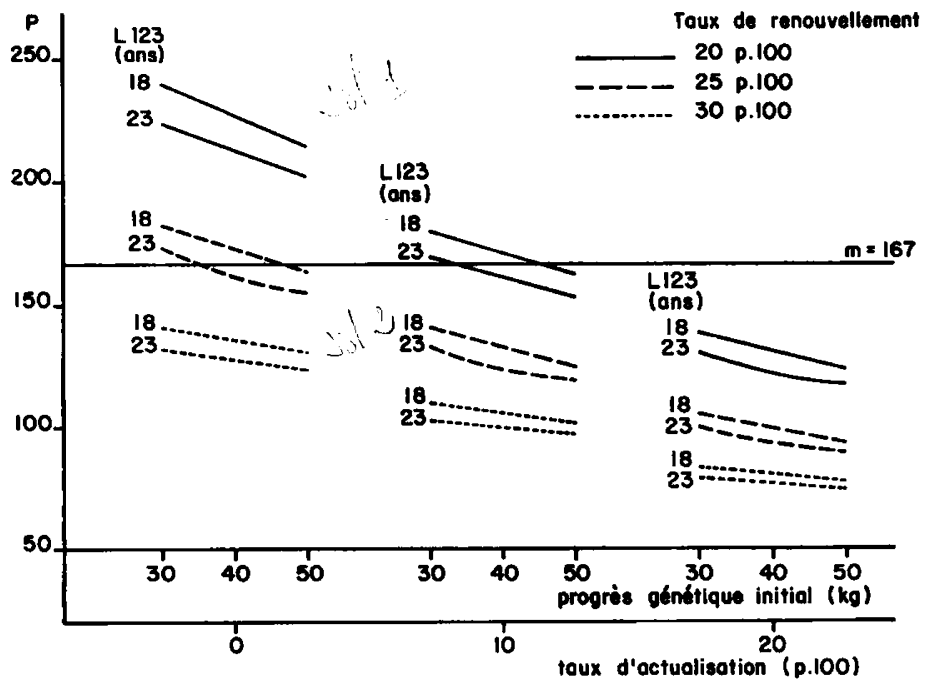

FIG. 3. - Rapport $P$ des pertes en lait aux gains en nombre de veaux croisés issus de mâles de grand format en fonction des variables envisagés

Ratio $P$ of milk loss to gain in number of crossed calves from large-sized males as related to the variables proposed

$\mathrm{L}_{123}$ est la somme des intervalles de génération des voies autres que la voie mères-filles.

Le rapport $P=\frac{L_{1}+L_{2}}{V}$ où $L_{1}$ et $L_{2}$ sont les pertes totales actualisées cumulatives et non curnulatives exprimées en $\mathrm{kg}$ de lait et $\mathrm{V}$ le gain total actualisé exprimé en nombres de veaux croisés issus de mâles de grand format.

$m=167$ est un exemple de rapport de marges (voir texte).

D'une manière générale $P$ varie de 60 à 240 entre les situations extrêmes étudiées, et les facteurs de variation les plus importants sont le taux de renouvellement et le taux d'actualisation. 
La première solution a ainsi d'autant moins de chances d'être préférable que l'on pénalise le long terme par un taux d'actualisation élevé et d'autant plus de chance de l'être que le taux de renouvellement est faible donc que l'on pratique davantage de croisement. En particulier si la valeur de $m$ est inférieure à roo le croisement des jeunes femelles non sélectionnées pour le renouvellement est préférable dans presque tous les cas; au contraire pour des valeurs supérieures à 200 on aura presque toujours intérêt à ne croiser que des femelles adultes.

En prenant comme type de mâles de croisement, les mâles de race Limousine pour les jeunes femelles et de race Charolaise pour les adultes, on peut ainsi penser que dans les conditions françaises où la différence de valeur des veaux croisés de 8 jours varie entre $5^{\circ}$ et $\mathrm{I}_{50} \mathrm{~F}$ selon les régions et où la marge du litre de lait est certainement inférieure à $0,30 \mathrm{~F}$ que le rapport des prix sera favorable à la seconde solution.

\section{III. - DISCUSSION - CONCLUSION}

Comme dans toute étude de prévision, les résultats que nous avons obtenus ici sont fortement dépendants de nos hypothèses de départ. L'importance des effets de mise en place dans la diminution du progrès génétique en lait (65 à $75 \mathrm{p}$. IOo) est liée au fait d'avoir choisi comme situation initiale celle d'une population où une partie des jeunes femelles est croisée. Bien que cette situation nous ait paru la plus vraisemblable d'autres hypothèses auraient pu être adoptées, revalorisant ou non l'importance du décalage initial, donc l'intérêt de la première politique.

Ainsi, si on prend pour comparer les deux politiques à l'équilibre le critère :

$$
\frac{\left(\Delta g^{\prime}-\Delta g^{\prime \prime}\right) \sum_{t=1}^{\mathrm{T}} \mathrm{d}^{t}}{\mathrm{P}_{c}\left(\left(\mathrm{I}-q_{1}^{\prime}\right) \alpha_{1}+\left(\mathrm{I}-q_{2}^{\prime}\right) \alpha_{2}\right)}
$$

on trouve, avec un taux d'actualisation de ro $\mathrm{p}$. roo, des valeurs de la variable $\mathbf{P}$ comprises entre 70 et $\mathrm{I} 70$ (contre 97 à $\mathrm{I} 80$ ), l'influence des différents paramètres (progrès génétique initial, taux de renouvellement) restant les mêmes. On remarquera que le choix de la deuxième politique (toutes les filles des jeunes femelles retenues pour le renouvellement) comme situation initiale ne change pas les résultats puisque les valeurs absolues du décalage initial $I$, des progrès génétiques $\Delta g^{\prime}$ et $\Delta g^{\prime \prime}$ et du pourcentage de veaux croisés $\left(I-q_{1}^{\prime}\right) \alpha_{1}+\left(I-q_{2}^{\prime}\right) \alpha_{2}$ sont identiques, seuls les signes étant inversés.

Enfin, on notera que notre objet n'était ici que l'étude d'un aspect marginal du problème plus vaste de l'intérêt du croisement terminal sur une population de femelles mixtes ou laitières, et qu'il conviendrait de discuter nos résultats et nos hypothèses dans ce cadre plus général. En effet, si, à la suite d'ANDERSson et LINDHE (I972), nous avons supposé qu'aucune élimination n'intervenait dans les élevages, le seul choix pratiqué étant un choix des génisses sur ascendance, il est certain que d'autres situations, sub-optimales du point de vue des critères utilisés dans toutes ces études mais sans doute plus proche de ce qui est réellement pratiqué, auraient pu être pris en compte : élimination des moins bonnes laitières après leur première 
ou deuxième lactation, croisement de toutes les femelles non encore connues sur leur propre performance avec des mâles de race à viande... Il faudrait alors tenir compte d'un certain nombre de facteurs que nous avons supposés indépendants des résultats tels que le coût de remplacement des femelles, l'effet de l'âge sur le niveau de production laitière... et ne plus raisonner seulement en espérance, mais également tenir compte des aléas du taux de masculinité ainsi que du risque de retenir pour la reproduction une fille de très mauvaise laitière, puisqu'un des intérêts du croisement systématique des génisses est précisément l'élimination de ce risque.

Reçu pour publication en mai 1976.

\section{REMERCIEMENTS}

Nous remercions L. Ollivier (CNRZ, Jouy en Josas, France) et W. Schlote (Hohenheim, RFA) pour la lecture critique du manuscrit.

\section{SUMMARY}

\section{OPTIMIZATION OF FEMALE REPLACEMENT}

AND TERMINAI, CROSSING IN DAIRY HERDS

Two methods of choosing dairy females for crossing are compared in a population of dairy cows having a minimum replacement rate. Maximum genetic progress in milk yield may be obtained with the first method discussed because only females with a high dairy index are used for replacement. However, to avoid calving difficulties smaller beef bulls must be used on the young females which are crossed.

In the second method, maximal crossing opportunities are used because terminal crossing is practised only on older females, using large-sized bulls, while all the daughters of young females are used for replacement.

These two methods are compared for the discounted profits obtained from the sale of crossed and pure calves and from the extra milk yield due to genetic progress. Various values of replacement rate, annual genetic progress in milk yield, generation interval and discounting rate are compared, taking as a reference the first method of choosing females.

Besides the advantage of the way itself for computing returns, it has been applied to each combination of the values proposed for the parameters to work out tables directly providing any comparison for given milk and meat prices.

Considering actual price conditions in France, the second solution seems better although difficult to put into practise.

\section{RÉFÉRENCES BIBLIOGRAPHIQUES}

Andersson J., Lindhe B., 1972. Optimum use of beef semen in a dual purpose or dairy breed. Journ. Fed. Eur. Zootech., Commission bovine et génétique, oct. 1972, Vérone (Italie), pp. 15.

Bichard M., Pease A. H. R., Swales Ph., Özü̈tüK K., I973. Selection in a population with overlapping generations. Anim. Prod., 17, 215-227.

Colleau J.-J., Poutous M., r973. Méthode de calcul des index de production laitière des femelles en France. Ann. Génét. Sél. anim., 5, 73-82.

Cunningham E. P., McClintock A. E., 1974. Selection in dual purpose cattle populations : effect of beef crossing and cow replacement rate. Ann. génét. Sel. anim., 6, 227-239. 
ELsen J.-M., Mocovor J.-C., r974. Recherches pour une rationalisation technique et economique des schémas de sélection des bovins et ovins. Bull. Tech. Dép. Génét. Anim. (Inst. nat. Rech. agron., Fr.), no r7.

Hıl W. G., I974. Prediction and evaluation of response to selection with overlapping generations. Anim. Prod., 18, II7-I39.

Rendel J.-M., Robertson A., 1950. Estimation of genetic gain in milk yield by selection in a closed herd of dairy cattle. J. Genet., 50, I-8.

Syrstad O., 1972. Effects of intensive culling in dairy herds. Acta. Agric. Scand., 22, 25-28. 